\title{
通信网络设计: 传输容量、设计代价和可扩展性
}

\author{
张国强 ${ }^{(1) *}$ ，张国清 ${ }^{(2)}$ \\ (1) 南京师范大学计算机科学与技术学院, 南京 210024 \\ (2) 中国科学院计算技术研究所, 北京 100190 \\ * 通信作者. E-mail: guoqiang@ict.ac.cn
}

收稿日期: 2010-03-17; 接受日期: 2010-10-19

国家自然科学基金 (批准号: 61100178, 61020106002) 和南京师范大学高层次引进人才科研启动基金 (批准号: 2011119XGQ0248) 资助项目

摘要 虽然目前围绕复杂网络的流量动力学研究日益增多, 但这类研究尚存在一些共性问题. 例如: 所采用的网络流模型过于简单; 没有考虑网络设计的代价和可扩展性. 本文建立在一个更为真实的 网络流模型之上, 同时为网络设计问题提供了更全面的视角. 本文在广为研究的传输容量之外又引 入了另外两个设计目标: 网络设计代价和可扩展性. 通过引入笛卡尔坐标系统, 本文量化分析了不同 的网络设计因素是如何独立地影响以及通过相互作用共同影响这些网络设计目标的. 结果表明, 不 同的网络拓扑结构呈现出不同的网络设计可行解空间形状, 并在实现高效费比和可扩展的网络设计 目标上表现出不同的能力. 特别地, 文章指出现有的经验性网络设计背后隐藏的理念不能满足高效 费比和可扩展的网络设计需求, 并提出了针对 $\mathrm{BA}$ 网络的一种高效费比和可扩展的设计方案, 即有 效路由结合基于有效介数的链路带宽分配方案. 而如果从头开始设计一个全新的网络, 则随机网络 是达成上述目标的最佳选择.

关键词 高效费比 通信网络设计网络传输容量 网络拓扑低成本信息化下一代网络 可扩 展网络设计

\section{1 引言}

随着数据密集型的新型应用以及对数据业务有需求的人群的迅速增长, 许多专家均承认现有的网 络基础设施已严重超负荷运转, 并接近其承载能力极限 ${ }^{[1,2]}$. 因此, 建立新的下一代数据网络已经迫在 眉睫.

然而, 虽然在网络设计领域已经有众多的工程技术用于解决各种网络设计中的具体问题, 并且基 于复杂网络视角的流量动力学研究也日益增长, 至今依然缺乏从宏观角度出发的网络设计理论 ${ }^{[3]}$. 随 着近十年来网络测量和建模研究的兴起 ${ }^{[4 \sim 8]}$, 这一问题变得尤为严重. 最近的研究表明: 许多实证网 络 (包括 Internet) 都呈现复杂的网络结构, 而非先前所假定的随机结构 [4,5,9 16]. 这一发现对网络设 计科学提出了新的需求. 美国自然科学基金委的网络科学和工程 (NetSE) 委员会提议把建立 “一种能 为大规模、高可靠、高效费比、可演化的网络系统设计提供系统的工程方法支撑的基础性科学” 作为 网络研究领域最主要的长远计划之一 ${ }^{[3]}$. 
建立这一基础性科学的主要难点在于网络设计问题的抽象化表示. 过于简单的模型会丧失它的实 用性, 而相反, 如果过于纠缠于细节, 则会迷失于细节而无法从宏观角度给出解决方案. 网络设计方法 的核心内容至少应包括下述基本问题的解决: 网络设计目标是什么? 网络设计元素是什么? 这些网络 设计元素之间是如何互作用的? 这些网络设计元素以及它们之间的相互作用是如何影响不同的网络 设计目标的? 最后, 这些设计目标的可行空间是什么? 从宏观角度来看, 网络设计通常包含一些相互 独立但又密切相关的方面. 其中有 3 个设计因素至关重要: 网络拓扑结构的设计、路由算法的选择以 及链路带宽的确定. 由于这些设计因素概念上相互正交, 因此对每种设计因素, 设计人员都可以独立 做出决定. 但是, 从达成网络设计目标的角度看, 这些设计因素实则紧密相关, 因为不同的选择组合对 设计目标的实现有不同的效果.

近年来, 研究界围绕复杂网络上的流量动力学展开了许多研究, 大部分旨在提高网络的传输容

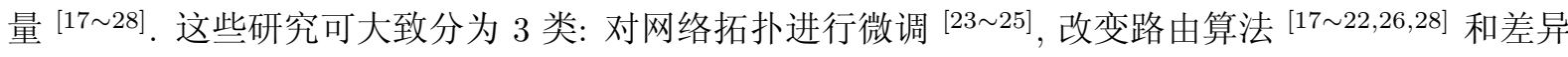
化节点处理能力 ${ }^{[27]}$. 提高网络传输容量最直接的途径是在网络中创建一些新的连接 ${ }^{[23,24]}$. 与之相 反, 文献 [25] 提出了一个反直觉的做法, 即在 BA 网络中通过删除一些连接高介数节点的边来达到均 衡网络负载从而提高网络传输容量的目标. 在流量动力学研究中获得最多关注的则是设计更高效的路 由算法. 这些算法包括仅依赖于拓扑信息的静态路由算法 (如中心避免算法 ${ }^{[18,26]}$ ) 以及同时考虑了网

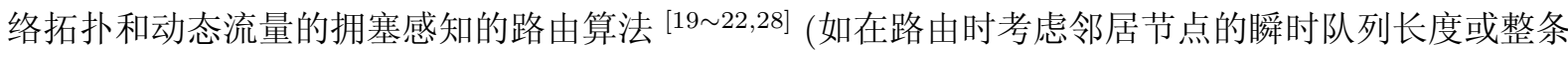
路径的队列长度之和). 文献 [27] 则提出了通过差异化节点的传输能力来提高网络传输容量的方法, 具 体包括两种节点能力分配模型, 分别是节点处理能力与节点度和节点介数成正比.

但是, 虽然这些研究具有启发意义, 它们依然存在一些共性问题: (1) 所有研究均专注于提高网络 传输容量, 而不考虑设计代价. 但在现实中, 网络设计通常受制于经济或技术因素; (2) 大部分研究都 只关注于某一个设计因素, 缺乏对不同设计因素及它们之间关系的全盘考虑; (3) 这些研究大都基于较 为简单的网络流模型, 与实际网络工程相去甚远; (4) 缺乏对网络设计方案可扩展性的研究.

我们之前的文章提出了通信网络设计的效费比问题 ${ }^{[29]}$, 探讨了在节点能力之和为定值的前提下 不同的节点能力分配方案对网络设计的影响, 这本质上将网络节点能力分配问题转变成了一个最优化 问题. 基于上述研究, 本文在下述几个方面进行了扩展:

- 对网络流模型进行了修改, 着重考虑链路带宽分配问题而非节点能力分配. 这是基于对网络设 计的如下两点认识: 1) 链路带宽 (而非节点的 CPU 处理能力) 的不足是通信网络产生拥塞的主要原 因; 2) 路由器包含多个端口, 每个端口都有自己独立的缓存. 拥塞发生在端口级别, 也就是说可能一个 路由器的某些端口拥塞了, 但其他端口却未拥塞.

- 在上述模型下, 理论分析了网络设计目标的可行空间, 对网络设计问题给出了更全面的视角.

- 在该模型下, 研究了网络传输容量和网络设计代价在不同的网络设计方案下的可扩展性.

- 增加了对真实 ISP 的路由器级拓扑以及真实路由协议的探讨.

特别地, 我们发现不同的网络在我们所定义的笛卡尔坐标空间中具有不同的设计目标可行空间形 状, 表明它们在实现高效费比的网络设计上所呈现的能力也不一样. 研究结果表明, 现有的经验性通 信网络设计理念可以大致被归类为 $(\mathrm{BC}, \mathrm{SPR})$. 这一设计方案在具有异构拓扑的网络上既不具备高 效费比, 也不具备可扩展性. 针对类似 BA 的无标度网络, 我们提出了一种兼具高效费比和高可扩展 性的设计方案, 即有效路由结合基于有效介数的带宽分配方案. 同时, 我们还发现在许多场合下, 随机 网络是实现上述两个设计目标的合适选择. 针对真实 ISP 路由器级拓扑的研究表明它们与 HOT 模型 相像, 而现有的 OSPF 路由中的权重设置策略较最短跳数路由更倾向于聚合流量. 


\section{2 网络流模型}

本文使用不同于文献 [25 30] 的网络流模型, 以更准确地模拟路由器的行为. 在本文的模型中, 路 由器包含多个端口, 每个端口都有独立的发送缓存队列. 每个端口的传输速率受限于它的带宽. 当一 个中间节点接收到数据包后, 它将数据包转发到相应的出口队列. 数据包在该队列等待传输. 拥塞发 生在端口级, 而不是路由器级. 可能一个路由器的某些端口发生了拥塞, 但其他端口并未拥塞.

在该模型中, 每个节点都能产生、转发和接收数据包. 每个时间步, 网络中都产生 $R$ 个数据包. 这些数据包具有随机的源和目标地址. 与之前为每个路由器赋一个唯一的节点能力不同, 我们为节点 上的不同端口赋不同的链路带宽. 记 $C\left(v_{i}\right)$ 表示节点 $v$ 的第 $i$ 个端口的带宽, 那么 $C\left(v_{i}\right)$ 给出了该端 口每个时间步能传输的最大数据包个数. 我们假设链路带宽是对称的, 即对每条边 $e=\left(v_{i}, u_{j}\right)$, 都有 $C\left(v_{i}\right)=C\left(u_{j}\right)$, 进一步记为 $C(e)$. 数据包依据特定的路由算法被逐跳转发到目的地. 当到达的和新创 建的数据包在端口 $v_{i}$ 累积超过 $C\left(v_{i}\right)$ 时, 数据包将在该端口的发送缓存排队, 并以先进先出 (FIFO) 的方式在随后的时间步得到处理. 如果存在多条备选路径, 则随机选择一条. 数据包抵达目的地后将 从系统中删除. 和文献 [25 29] 类似, 由于端口的发送队列长度与拥塞是否会发生无关, 我们将队列长 度设为无限大.

当 $R$ 从 0 开始增长, 可以预期存在一个由自由态至拥塞态的相变过程. 在自由态, 网络中平均产 生的数据包个数等于递交到目标节点的数据包个数; 而在拥塞态, 新产生的数据包个数超过了网络能 及时处理的数据包个数. 我们将相变产生时的临界数据包产生速率记为 $R_{c}$.

为了度量 $R_{c}$, 我们可以使用文献 [31] 提出的序参量 $\eta=\lim _{t \rightarrow \infty} \frac{\langle\Delta(\Theta)\rangle}{R \Delta t}$, 其中 $\Theta(t)$ 表示网络中 $t$ 时刻的数据包总数, $\Delta \Theta=\Theta(t+\Delta t)-\Theta(t),\langle\cdots\rangle$ 表示 $\Delta(t)$ 窗口时间的平均值. 当 $R<R_{c}$, 网络处于 自由态, 此时 $\Delta \Theta \approx 0$, 且 $\eta=0$; 而当 $R>R_{c}, \Delta \Theta$ 随着 $\Delta(t)$ 的增加而增加, 使得 $\eta>0$. 因此, 我们 可以通过仿真实验, 把 $\eta$ 从 0 开始发散时的数据包产生率作为 $R_{c}$.

\section{3 网络设计目标和网络设计因素}

本节我们给出通信网络设计人员最需要关注的网络设计目标和设计因素.

\section{1 网络设计目标}

一个网络设计总是具有明确的设计目标. 在本文中, 我们考察 3 个主要的设计目标: 网络传输容 量、设计代价和可扩展性.

提高网络传输容量是网络设计和工程领域最主要的目标之一, 可以通过临界数据包产生速率 $R_{c}$ 来衡量 ${ }^{[18,25 \sim 29]}$. 在基于最短路径算法的路由中, 介数 (betweenness) ${ }^{[30,32 \sim 35]}$ 能精确地估计经过每个 节点的流量, 从而能用于从理论上计算 $R_{c}$.

在我们的模型中, 对任意网络, 如果采用最短路径路由, 则每个时间步经过边 $e=\left(v_{i}, u_{j}\right)$ 的数据 包个数的期望值为 $\frac{R B(e)}{N(N-1)}, B(e)$ 表示边 $e$ 的边介数. 这些数据包中, 其中一半是从 $v_{i}$ 流向 $u_{j}$, 另一 半是从 $u_{j}$ 流向 $v_{i}$. 因此, 为了使端口 $v_{i}$ 不拥塞, 应满足 $\frac{1}{2} \times \frac{R B(e)}{N(N-1)} \leqslant C\left(v_{i}\right)$, 即 $R \leqslant \frac{2 C\left(v_{i}\right) N(N-1)}{B(e)}$. 从 而, 临界数据包产生速率 $R_{c}$ 为

$$
R_{c}=\min _{e \in E} \frac{2 C(e) N(N-1)}{B(e)},
$$

其中 $E$ 是网络的边集. 
在更一般的情形下, 对任何基于拓扑 ${ }^{1}$ ) 的路由算法 $\Gamma$, 我们引入有效边介数 (类似于文献 $\left.[25]\right) B^{\Gamma}(e)$ 来估算在路由算法 $\Gamma$ 下, 每个时间步经过一条边 $e$ 的数据包个数的期望值, 具体定义如下:

$$
B^{\Gamma}(e)=\sum_{u, v \in V, u \neq v} \frac{\delta_{(e)}^{\Gamma}(u, v)}{\delta^{\Gamma}(u, v)},
$$

其中, $V$ 是节点集, $\delta^{\Gamma}$ 表示在路由算法 $\Gamma$ 下, 节点 $u, v$ 之间的备选路径条数, 而 $\delta_{(e)}^{\Gamma}$ 则表示这些备选 路径中经过边 $e$ 的路径条数.

根据该定义, 在路由算法 $\Gamma$ 下的临界数据包产生速率 $R_{c}$ 可以通过如下公式计算:

$$
R_{c}=\min _{e \in E} \frac{2 C(e) N(N-1)}{B^{\Gamma}(e)} .
$$

这一公式同时也回答了不同的设计因素如何影响其中的一个网络设计目标 $\left(R_{c}\right)$ 的问题. $C(e)$ 代 表了链路带宽分配策略对 $R_{c}$ 的影响, 而 $B^{\Gamma}(e)$ 则综合了网络拓扑和路由算法对 $R_{c}$ 的影响.

网络设计代价是第二个网络设计目标, 可以用不同的方式来度量, 通常关系到经济或技术问题. 如 果能以较低的设计代价实现同样的网络传输容量, 则有助于实现低成本信息化. 由于我们将链路带宽 之和设为定值, 以比较不同的链路带宽分配策略, 因此, 一个合理的度量网络设计代价的目标函数为最 大带宽 $C_{\max }$. 这是因为它直接关系到给定的设计方案在技术上是否具有可行性. $C_{\max }$ 给出了一个设 计方案的链路带宽上限值, 从而可以用于判断该设计方案是否可用现有的技术能力来实现.

可扩展性是本文探讨的最后一个设计目标. 由于现在的网络都具有大规模的特性, 并且处于不断 的演化过程中, 可扩展性也变得愈来愈重要. 一个可扩展的网络设计方案无论对网络投资者或运营者 都具有长期的好处. 在本文中, 我们通过 $R_{c}$ 和 $C_{\max }$ 的增长趋势来度量一个网络设计方案的可扩 展性.

\section{2 网络设计因素}

路由算法、链路带宽分配策略和网络拓扑是 3 个关键的网络设计因素. 虽然流量需求 (通常以流 量矩阵来表示) 也是网络设计中的一个重要因素, 但它会随着时间变化而变化, 因此我们在本文不考 虑流量需求矩阵. 设计人员所能采用的设计因素可能会因时因地而不同. 例如, 当从头设计一个全新 的网络时, 设计人员可以对任何设计因素都自由选择; 而当提升现有网络时, 设计人员只能够改变路 由算法或升级链路带宽.

虽然拥塞感知的动态路由算法可以在一定程度上提高网络传输容量、避免拥塞, 但众所周知它们 也会引起路由振荡 ${ }^{[36,37]}$. 因此, 本文我们仅考虑静态路由. 我们考虑了两种路由算法: 一种是最短路 径路由, 简写为 SPR; 另一种是中心避免路由算法之一, 称为有效路由算法 ${ }^{[26]}$, 简写为 EFR. 更精确 地, EFR 在节点 $s$ 和 $t$ 之间选择一条路径 $s=v_{0}, v_{1}, \ldots, v_{k}=t$, 使得目标函数 $\sum_{0 \leqslant i<k} d\left(v_{i}\right)$ 最小, 其 中 $d\left(v_{i}\right)$ 表示节点 $v_{i}$ 的度. 选择 EFR 是因为它易于实现, 并且不需要对 Dijkstra 算法进行修改, 同时 它又能获得非常好的性能. 文献 [18] 提出了另一个称为 HAP 的中心避免路由算法, 但其分布式实现 过于复杂.

本文考察了 3 种链路带宽分配策略: 均匀带宽分配策略、基于介数带宽分配策略和基于有效介数 的带宽分配策略, 分别简记为 UC, BC 和 EBC. 在 UC 中, 每条链路都具有相同的带宽; 而在 BC 和 $\mathrm{EBC}$ 中, 每条链路的带宽分别与它的边介数和有效边介数成正比. 为了将带宽分配描述为一个最优化

1) 基于拓扑的路由算法指路由决策仅基于静态拓扑信息, 而不基于动态流量信息 
表 15 种网络的基本特征

Table 1 The basic properties of five kinds of networks a)

\begin{tabular}{ccccc}
\hline Network & $N$ & $M$ & $D$ & $L$ \\
\hline WS & 1200 & 2400 & 15.5 & 7.86 \\
ER & 1200 & 2450 & 11 & 5.23 \\
BA & 1200 & 2390 & 8 & 4.43 \\
PA & 1200 & 2400 & 8.7 & 4.03 \\
HOT & 1200 & 2583 & 9 & 5.16 \\
\hline
\end{tabular}

a) $N$ and $M$ represent the number of nodes and edges respectively, $D$ is the diameter, and $L$ is the average shortest path length

问题, 我们将带宽之和设为常数, 这里设置为 $\sum_{e} B(e)=M$. 文献 [27] 也探讨了基于 $\mathrm{BC}$ 的策略, 但 它是针对节点能力的, 并且也没有将其作为优化问题来对待.

我们探讨了 5 种具有代表性的网络拓扑结构: $\mathrm{BA}^{[4]} 、 \mathrm{HOT}^{[38]} 、 \mathrm{ER}^{[39]} 、 \mathrm{WS}^{[40]}$ 和 $\mathrm{PA}^{[30]}$. $\mathrm{PA}$ 是 $\mathrm{BA}$ 模型的变种, 它首先利用 $\mathrm{BA}$ 模型构建一棵树状的网络 $(m=1)$, 然后在网络中增加 $N$ 条内部边. 每条内部边按下述方式选择, 即它的两个端点被选择的概率均与其节点度成正比. HOT 模型是针对 Internet 路由器级拓扑的启发式模型, 其中的节点可分为 3 个层次: 构成核心网的节点度较低的核心 路由器, 与核心路由器直接相连的高节点度的接入网路由器, 以及与接入网路由器直接相连的低节点 度的边缘路由器. 本文使用的 HOT 网络具有与相应的 PA 网络相似的节点度分布. 表 1 给出了这些 网络的基本网络特征.

\section{4 笛卡尔坐标系统和可行空间}

本节理论分析了在我们的网络流模型下, 给定网络的设计目标可行解空间.

\section{1 笛卡尔坐标系统}

对于给定网络 $G$, 针对 $C_{\max }$ 和 $R_{c}$, 一个网络设计方案的效果可以映射到一个二维笛卡尔空间的 一个坐标. 该二维笛卡尔空间以 $C_{\max }$ 作为 $X$ 坐标轴, 以 $R_{c}$ 作为 $Y$ 坐标轴. 那么, 最自然的问题是 $C_{\text {max }}$ 和 $R_{c}$ 的取值范围是什么?

$C_{\max }$ 的取值范围显然是 $[1, M]$, 最优 (最小) 值在均匀带宽分配策略时达到, 而最差 (最大) 值则 是将所有的带宽都分配给一条边.

对于 $R_{c}$, 下界为 0 , 例如当路由算法让数据包在网络中不断循环. 而 $R_{c}$ 的上界则可以由如下定理 给出:

定理 1 给定网络 $G$, 任何网络设计方案所能达到的 $R_{c}$ 上界为 $2 M / L$, 这里 $L$ 是 $G$ 的平均最短 路径长度; 同时, 这一上界当且仅当使用 $(\mathrm{BC}, \mathrm{SPR})$ 时达到.

证明 由于 $\sum_{e} B(e)=M$, 端口的带宽之和为 $2 M$. 这表明网络每个时间步最多可以转发 $2 M$ 个 数据包. 由于每个数据包都具有随机的源和目的地址, 在路由算法 $\Gamma$ 下, 平均每个包从源到目的地的 路径长度为 $L^{\Gamma}$, 这意味着网络平均每个时间步至多消耗 $2 M / L^{\Gamma}$ 个数据包. 因此, 依据 Little 定律, 有

$$
R_{c} \leqslant 2 M / L^{\Gamma} \leqslant 2 M / L
$$


这证明了 $2 M / L$ 是 $R_{c}$ 的一个上界. 下面我们证明这一上界当且仅当应用 (BC, SPR) 方案时达 到. 首先, 我们证明应用 $(\mathrm{BC}, \mathrm{SPR})$ 是达到最大 $R_{c}$ 的充分条件. 实际上, 当采用该方案时, $R_{c}$ 可以重 写为

$$
\begin{aligned}
R_{c} & =\min _{e \in E} \frac{2 C(e) N(N-1)}{B(e)}=\min _{e \in E} \frac{2 B(e) / \sum_{f \in E} B(f) \times M \times N(N-1)}{B(e)} \\
& =\frac{2 M N(N-1)}{\sum_{f \in E} B(f)}=\frac{2 M N(N-1)}{N(N-1) L}=\frac{2 M}{L} .
\end{aligned}
$$

其次我们证明应用 (BC, SPR) 是达到最大 $R_{c}$ 的必要条件. 根据不等式 (4), $R_{c}$ 达到 $2 M / L$ 当且仅当 下列两个条件同时成立: 1) $L^{\Gamma}=L$, 表明路由算法为 $\left.\mathrm{SPR} ; 2\right)$ 平均每个时间步网络都移动 $2 M$ 个数据 包到下一跳. 由于在 SPR 中, 在自由态时平均每个时间步到达端口 $v_{i}$ 的数据包个数恰好为 $\frac{1}{2} \frac{R B(e)}{N(N-1)}$, 这里 $e$ 是端口 $v_{i}$ 的关联边. 当 $R=2 M / L$ 时, 网络中的平均数据包个数为 $\sum_{e} \frac{R B(e)}{N(N-1)}=2 M$. 因此, 为了使得整个网络每个时间步能移动 $2 M$ 个数据包, 每个端口在每个时间步应恰好移动 $\frac{M B(e)}{N(N-1) L}$ 个 数据包, 这正对应了基于 $\mathrm{BC}$ 的链路带宽分配策略. 这表明 (BC, $\mathrm{SPR}$ ) 是达到 $R_{c}$ 上限的唯一方案. 证毕.

事实上, $(\mathrm{BC}, \mathrm{SPR})$ 设计方案折射了现实中经验性的网络设计理念. 现实的网络路由协议如 OSPF 和 BGP 通常基于最短路径路由 (或至少在路径选择时将路径长度作为一个主要决策因素). 高带宽的 链路通常布置在网络中的关键位置, 当一些链路超载时, 就需要进行带宽升级, 这可能会吸引更多的 流量, 从而在流量需求和带宽升级之间形成恶性循环.

定理 1 同时表明除了完全同质的规则网络, $R_{c}$ 和 $C_{\max }$ 的最优值无法同时达到, 这表明在这两个 设计目标之间存在折中问题. 表 2 和表 3 分别给出了 $R_{c}$ 的理论值和仿真结果, 而表 4 则给出了 $C_{\max }$ 的值. 可以看到, 虽然 (BC, SPR) 可以达到最大的 $R_{c}$, 它通常都引起较大的 $C_{\max }$, 因为 $C_{\max }$ 与最大 边介数成正比, 而在异构网络中最大边介数的值很大. 那么, 一个自然的问题是, 当 $C_{\max }$ 取最小值时, $R_{c}$ 的取值范围是什么?

当应用 (UC, SPR) 时, 很容易证明 $R_{c}=\frac{2 N(N-1)}{B_{\max }}$. 在异构网络中, $B_{\max }$ 可以非常大, 导致 $R_{c}$ 很 小, 远低于 (BC, SPR) 方案能达到的最优值 $2 M / L$ (见图 1(a)). 对类似 BA 的网络, 可以通过改变路

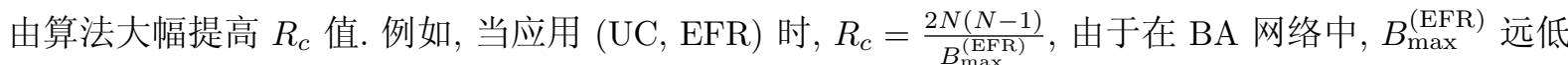
于 $B_{\max }$, 因此改变路由算法可以大幅提高 BA 网络的传输容量. 但是, 对 HOT 模型和小世界模型的 网络, (UC, EFR) 基本无效.

很显然, 当 $C_{\max }$ 取最优值时, $R_{c}$ 也存在一个极大值, 但是寻找一个能达到这一极大值的实用路 由算法并不容易. 当应用 $\mathrm{UC}$ 时, $R_{c}=\frac{2 N(N-1)}{B_{\max }^{\Gamma}}$, 因此在这种情况下实现 $R_{c}$ 极大值的本质就是找到最 小化 $B_{\max }^{\Gamma}$ 的路由算法. 这等价于寻找一个大小为 $N(N-1)$ 的路径集, 每个节点对一条路径, 使得该 路径集中边出现的最大频次最小化. 这一路由算法可以通过下述贪婪算法来构建: 首先计算任何两点 之间的所有简单路径, 然后枚举所有满足条件的路径集并计算 $B_{\text {max }}^{\Gamma}$, 然后依据 $B_{\max }^{\Gamma}$ 最小的路径集构 造路由算法. 但是, 这一方法在现实中并不可行, 因为其计算复杂度是指数时间的. 因此, 本文仅指出 这一极大值的存在性. 这一设计方案的重要性在于它是确定可行解空间的一个关键坐标点.

图 2(a) 示例了对于 BA 网络, 其可行解空间的形状. 图中给出了本文探讨的 4 个典型设计方案对 应的坐标, 以及其他 3 个对于给出可行解的概念空间比较重要的坐标: $A, B$ 和 $C$. 其中, $A$ 代表 $\mathrm{UC}$ 和基于循环的路由算法, $B$ 表示将所有的带宽都分配给一条边的带宽分配策略, $C$ 表示基于 $\mathrm{UC}$ 的带 宽分配策略时取得极大 $R_{c}$ 的设计方案.

总体来说, $A, B, C$ 和 (BC, SPR) 4 个坐标粗略地勾勒出了可行解空间的轮廓. 但是, 对不同的网 
表 2 表格 1 中不同的网络在不同的链路带宽分配策略和路由算法组合下的理论 $R_{c}$ 值

Table 2 Theoretical result of the $R_{c}$ under different combinations for networks in Table $1^{\mathrm{a})}$

\begin{tabular}{crrrrr}
\hline (BS, RP) & BA & PA & HOT & ER & WS \\
\hline (UC, SPR $)$ & 88.5 & 92.8 & 99.1 & 284.5 & 111.1 \\
$($ UC, EFR $)$ & 264.3 & 195.3 & 94.6 & 390.6 & 147.9 \\
$($ BC, SPR $)$ & 1079.4 & 1192.2 & 1001.7 & 937.9 & 610.2 \\
$($ EBC, EFR $)$ & 766.6 & 844.9 & 909.2 & 891.4 & 606.2 \\
\hline
\end{tabular}

a) BS stands for bandwidth allocation scheme and RP stands for routing algorithm. The results are obtained from Eq. (3). The result presented is the average of the 10 realizations

表 3 BA, PA, HOT, ER 和 WS 网络在不同的链路带宽分配策略和路由算法组合下 $R_{c}$ 的仿真结果

Table 3 Simulation result of the critical packet-generating rate Rc under different combinations for BA, PA, HOT, ER and WS networks a)

\begin{tabular}{cccccc}
\hline (BS, RP) & BA & PA & HOT & ER & WS \\
\hline (UC, SPR) & 110.2 & 115.6 & 119.9 & 370.5 & 136.7 \\
(UC, EFR) & 320.4 & 249.6 & 114.6 & 519.8 & 174.7 \\
(BC, SPR) & 934.2 & 1042.7 & 840.8 & 844.2 & 517.0 \\
(EBC, EFR) & 678.0 & 741.0 & 768.2 & 809.9 & 519.9 \\
\hline
\end{tabular}

a) For each kind of network, 10 instances are generated and for each instance we run ten simulations. The result here is the average over all the simulations. Compared with Table 2, it is shown that the simulation result is roughly consistent with the theoretical analysis

\section{表 4 不同网络设计方案的 $C_{\max }$ 值, 其中缩写的含义与表 2 同}

Table $4 C_{\max }$ under different network designing schemes, where the meaning of acronyms is the same as Table 2

\begin{tabular}{crrrrc}
\hline$(\mathrm{BS}, \mathrm{RP})$ & BA & PA & HOT & ER & WS \\
\hline$\left(\mathrm{UC},{ }^{*}\right)$ & 1 & 1 & 1 & 1 & 1 \\
$\left(\mathrm{BC},{ }^{*}\right)$ & 12.43 & 12.99 & 10.30 & 3.37 & 5.63 \\
$(\mathrm{EBC}, \mathrm{EFR})$ & 2.91 & 4.45 & 9.70 & 2.40 & 4.13 \\
\hline
\end{tabular}

络, 可行解空间的形状可能截然不同. 例如, 一个规则网络的可行解空间看上去将类似图 2(b), 其中, 本文讨论的所有 4 个设计方案和 $C$ 都将收敛于同一个坐标 $C$.

\section{2 高效费比设计的存在性}

从工程的角度, 人们关心是否存在设计方案能在 $R_{c}$ 和 $C_{\max }$ 之间取得很好的折中效果, 从而以较 低的成本实现既定的目标. 这一问题的答案依赖于特定的网络拓扑. 在 BA 网络中, 本文提出的 (EBC, $\mathrm{EFR}$ ) 设计方案在这两个设计目标之间取得了很好的平衡, 其原因在于

$$
\frac{R_{c}^{(\mathrm{BC}, \mathrm{SPR})}}{R_{c}^{(\mathrm{EBC}, \mathrm{EFR})}}=\frac{L^{(\mathrm{EFR})}}{L}
$$



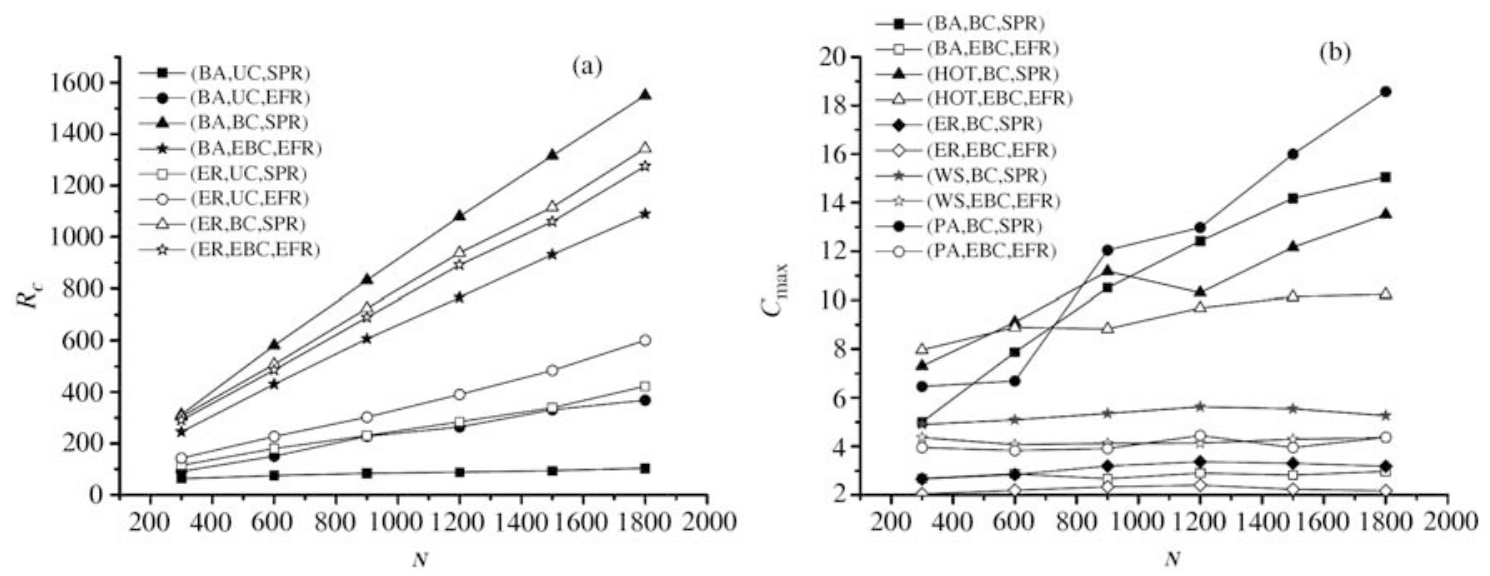

图 1 (a) 在 4 种链路带宽分配策略和路由算法的组合下, BA 和 ER 网络 $R_{c}$ 值的扩展性; (b) 分别采用 (BC, SPR) 和 (EBC, EFR) 时 5 种网络 $C_{\text {max }}$ 的扩展性

Figure 1 Scalability of (a) $R_{c}$ values of $\mathrm{BA}$ and ER under four different configurations and (b) $C_{\max }$ values of five networks under (BC, SPR) and (EBC, EFR) schemes
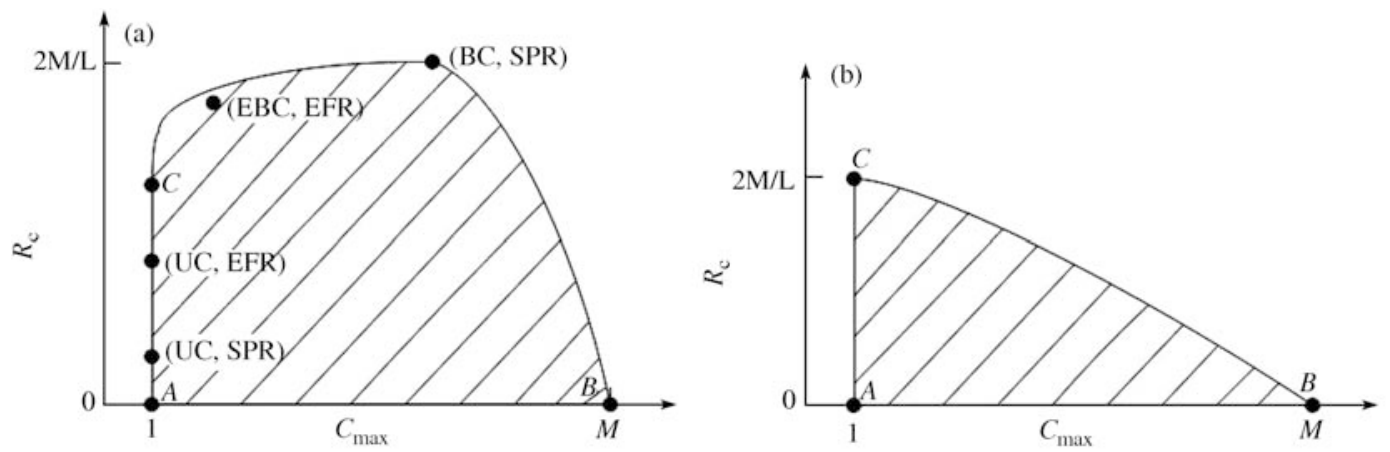

图 2 BA 和正则网络的设计可行解空间图示

Figure 2 Illustration of the designing space for (a) BA-like networks and (b) regular networks

及

$$
\frac{C_{\mathrm{max}}^{(\mathrm{BC}, \mathrm{SPR})}}{C_{\max }^{(\mathrm{EBC}, \mathrm{EFR})}}=\frac{L^{(\mathrm{EFR})}}{L} \times \frac{B_{\max }}{B_{\max }^{(\mathrm{EFR})}} .
$$

在许多网络中, $L^{(\mathrm{EFR})}$ 仅稍大于 $L(\text { 见图 3(b) })^{2)}$, 因此, $(\mathrm{EBC}, \mathrm{EFR})$ 方案的 $R_{c}$ 值略小于最优值.

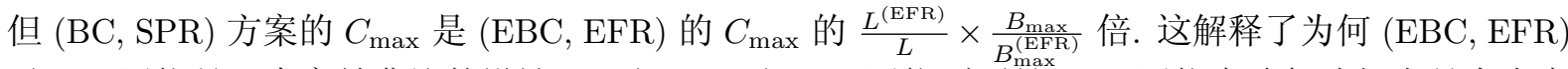
对 BA 网络是一个高效费比的设计, 而对 HOT 和 WS 网络则不是. BA 网络在路径选择上具有内在 的多样性, 对路由算法改变有很强的敏感性. 在这类网络中, $B_{\max }$ 可以是 $B_{\max }^{(\mathrm{EFR})}$ 的数量级倍数, 使得 (EBC, EFR) 的 $C_{\max }$ 与 $(\mathrm{BC}, \mathrm{SPR})$ 的 $C_{\max }$ 相比很小. 在 HOT 网络中, 虽然度分布相同, 但它严格 的层次结构限制了其路径选择的灵活性, 使它对路由算法不敏感, 因此 $B_{\max }$ 和 $B_{\text {max }}^{(\mathrm{EFR})}$ 区别很小. 在 $\mathrm{WS}$ 网络中, 近乎均匀分布的度分布使得 SPR 和 EFR 几乎效果相同, 因此 $B_{\max }$ 和 $B_{\max }^{(\mathrm{EFR})}$ 的区别也 很小. HOT 网络和 WS 网络的区别在于, 在 HOT 网络中, $C_{\max }$ 较高, 而在 WS 网络中, $C_{\max }$ 较小. $\mathrm{ER}$ 网络的随机结构使得它位于 WS 和 BA 网络之间. 对 ER 网络来说, (BC, SPR) 和 (EBC, EFR) 都是很好的设计选择. 与其他网络相比, 即便是 (UC, SPR) 和 (UC, EFR) 也可以获得相对较高的 $R_{c}$.

2) 这也保证了本文所讨论的所有网络的路径长度代价 (或者称为路径拉伸系数) 是可接受的 

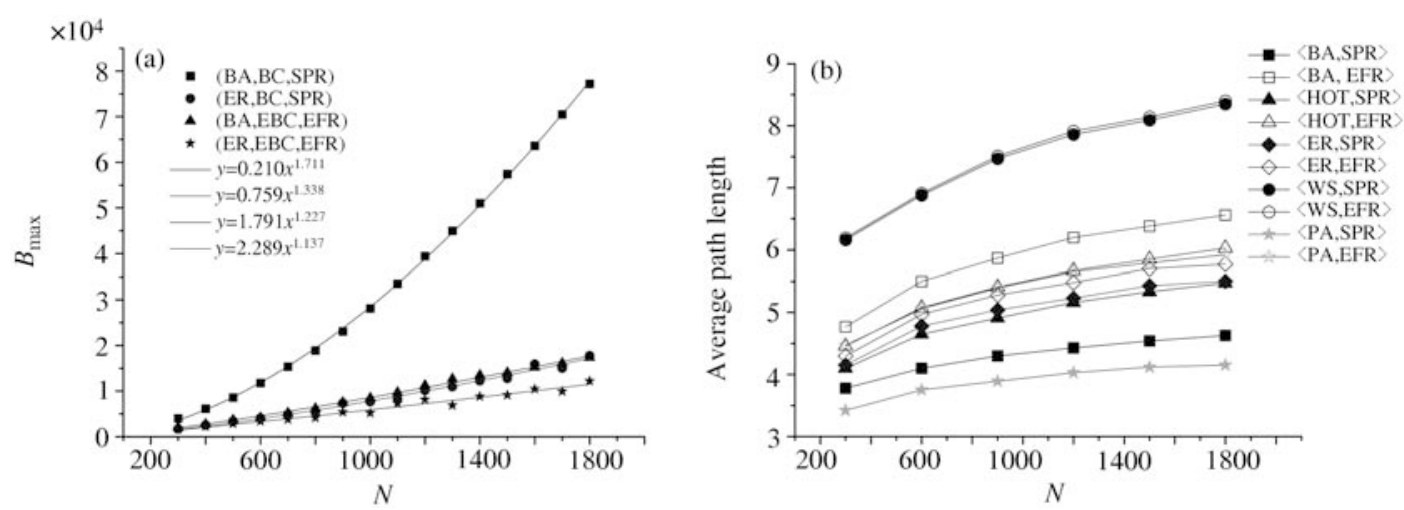

图 3 (a) BA 网络和 ER 网络的最大边介数和最大有效边介数的可扩展性; (b) SPR 和 EFR 路由算法下 5 种 不同规模网络的最短路径长度变化情况. 实心形状代表 SPR，而空心的形状代表 EFR

Figure 3 (a) Scalability of the maximal betweenness and effective betweenness for BA and ER networks, and the line fittings; (b) average path length for the five networks with different size under both SPR and EFR. The filled shape represents the SPR, while the hollow shape represents the EFR

\section{5 网络设计方案的可扩展性}

一个设计方案的可扩展性可以通过网络演化时 $R_{c}$ 和 $C_{\max }$ 的增长趋势来研究. 图 1 (a) 给出了 4 种不同的设计方案在不同规模的 BA 和 $\mathrm{ER}$ 网络上的 $R_{c}$ 值变化, 而图 1(b) 则给出了在分别采用 $(\mathrm{BC}$, $\mathrm{SPR}$ ) 和 (EBC, EFR) 两种策略时, 5 种网络的 $C_{\max }$ 值随网络大小的变化. 可以发现采用 (UC, SPR) 方案时, BA 网络的 $R_{c}$ 值非常稳定, 几乎与 $N$ 的规模无关. 这表明, $B_{\max }$ 随着 $N$ 呈超线性增长, 如 图 3(a) 所示. 数值拟合表明: 在 BA 网络中, $B_{\max } \sim N^{1.711}$; 而在 ER 网络中, $B_{\max } \sim N^{1.338}$. 另一方 面, 随网络规模的扩展, $B_{\text {max }}^{(\mathrm{EFR})}$ 增长相对缓慢, 在 $\mathrm{BA}$ 网络和 $\mathrm{ER}$ 网络中分别是 $\sim N^{1.227}$ 和 $\sim N^{1.137}$. 这表示, 应用 (UC, SPR) 方案时, BA 网络的 $R_{c}$ 增长缓慢, 而在 $\mathrm{ER}$ 网络中, $R_{c}$ 增长较快. 但应用 $(\mathrm{UC}, \mathrm{EFR}), R_{c}$ 在 $\mathrm{BA}$ 网络上的可扩展性得到了极大的提高.

对于 $C_{\max }$, 应用 (BC, SPR) 时, $C_{\max } \propto B_{\max }$, 因此它在 BA 网络中增长很快, 但在 $\mathrm{ER}$ 网络中增 长缓慢得多. 应用 $(\mathrm{EBC}, \mathrm{EFR})$ 时, $C_{\max } \propto B_{\max }^{(\mathrm{EFR})}$, 在 $\mathrm{BA}$ 网络中可扩展性很低. 这可以从图 $1(\mathrm{~b})$ 得 以验证, 可以看到在 $(\mathrm{BA}, \mathrm{BC}, \mathrm{SPR})$ 中, $C_{\max }$ 增长很快, 但在 (BA, EBC, EFR) 和 (ER, EBC, EFR) 中几乎保持稳定.

\section{6 现实意义}

虽然本文的重点是对不同网络设计因素如何影响网络设计目标进行理论分析, 我们希望分析的结 果可以用于指导未来通信网络设计. 为此, 我们需要对现有网络拓扑和路由算法有一个基本的认识. 本 节, 我们使用一些真实的由 Rocketfuel 测量的 ISP 路由器级互联网拓扑 [41] 来探讨真实路由器级拓扑 和路由算法的设计. 我们使用了 AS3967, AS1221 和 AS1239 的拓扑. 表 5 给出了这 3 个拓扑的大小, 表 6 给出了在不同的链路带宽分配策略和路由算法组合下 3 种网络的 $R_{c}$ 和 $C_{\max }$ 值. 与表 3 和表 4 相比, 我们发现真实网络拓扑与 HOT 网络类似. 注意到 HOT 网络的基本特征是: 1) 当使用均匀链路 带宽分配策略时, $R_{c}$ 一般都较小, 且对路由算法不敏感; 2) 高 $R_{c}$ 同时意味着高 $C_{\max }$, 即不存在高效 费比的设计. 这些特征在真实 ISP 拓扑中也被观测到了. 因此, 之前对于 HOT 网络的高效费比和可 
表 53 个真实 ISP 路由器级拓扑的规模

Table 5 Size of several real ISPs' router-level topologies

\begin{tabular}{cccc}
\hline & AS3967 & AS1221 & AS1239 \\
\hline$N$ & 353 & 2508 & 7282 \\
$M$ & 820 & 3027 & 9828 \\
\hline
\end{tabular}

表 63 个真实路由器级互联网拓扑上不同的链路带宽分配策略和路由算法组合下的 $R_{c}$ 和 $C_{\max }$ 值

Table $6 \quad R_{c}$ and $C_{\max }$ values of different combinations of link bandwidth allocation schemes and routing algorithms for several real ISPs' router-level Internet topologies

\begin{tabular}{crrrrrr}
\hline \multirow{2}{*}{$(\mathrm{BS}, \mathrm{RP})$} & \multicolumn{2}{c}{ AS3967 } & \multicolumn{2}{c}{ AS1221 } & \multicolumn{3}{c}{ AS1239 } \\
\cline { 2 - 7 } & \multicolumn{1}{c}{$R_{c}$} & $C_{\max }$ & $R_{c}$ & $C_{\max }$ & $R_{c}$ & $C_{\max }$ \\
\hline (UC, SPR) & 18.66 & 1.00 & 6.95 & 1.00 & 42.13 & 1.00 \\
$(\mathrm{UC}, \mathrm{EFR})$ & 13.63 & 1.00 & 6.63 & 1.00 & 51.56 & 1.00 \\
$(\mathrm{UC}, \mathrm{OSPF})$ & 4.63 & 1.00 & 7.49 & 1.00 & 17.07 & 1.00 \\
$(\mathrm{BC}, \mathrm{SPR})$ & 286.43 & 15.35 & 1029.07 & 148.12 & 3663.41 & 86.94 \\
$(\mathrm{EBC}, \mathrm{EFR})$ & 135.78 & 19.93 & 910.64 & 137.27 & 3236.14 & 62.76 \\
$(\mathrm{EBC}, \mathrm{OSPF})$ & 208.08 & 44.96 & 1020.85 & 136.28 & 3405.47 & 199.46 \\
\hline
\end{tabular}

扩展性的讨论也应该适用于路由器级互联网拓扑.

真实的互联网的域内路由通常采用两类典型的路由算法: RIP 和 OSPF. RIP 选择最小跳数的路 径, 本质上与本文的 SPR 一致. OSPF 算法为每条链路设置一个权重, 并选择权重之和最小的路径. 为了考察真实路由协议的性能, 我们在表 6 中同时也包含了 OSPF. 但是, 真实的链路权重很难推测, ISP 通常也不愿意公开这些信息. Rocketfuel 提供了核心网路由器之间链路的权重, 但并没有提供其 他链路的权重. 我们使用如下的简单启发式方法来为其他链路赋值权重. 首先, 我们将链路分为 3 类: 1) 接入路由器之间的链路; 2) 接入路由器和核心路由器之间的链路; 3) 核心路由器之间的链路. 我们 假设这 3 类链路之间的平均权重之比为 1:3:15, 这一假设与文献 [42] 的假设相同. 从表 6 我们可以看 到, 在应用 (EBC, OSPF) 时, AS3967 和 AS1239 的 $C_{\max }$ 值比应用 (BC, SPR) 时要大的多, 意味着当 前使用的权重赋值策略倾向于在网络中聚合流量. 当流量需求或网络规模增长时, 对 $C_{\max }$ 的需求会 更高, 这在实际中对应了频繁的链路带宽升级.

\section{7 结论}

在本文中, 我们对现有通信网络的流量模型做了修改, 使之包含了链路带宽分配问题, 从而克服了 先前许多网络流动力学研究中存在的模型太简单和不真实的问题. 此外, 我们提出了两个额外的网络 设计目标, 即设计代价和可扩展性. 文章在笛卡尔坐标系统的框架下分析了主要的设计因素是如何单 独和通过相互作用影响这些设计目标的, 结论表明不同的网络呈现出不同的解空间形状, 并且在允许 高效费比和可扩展设计上的表现也不同.

特别地, 我们发现当前广泛被接受的经验性网络设计和工程方法无法满足高效费比和可扩展的需 求, 无法实现低成本信息化的目标. 高效费比和高可扩展设计方案的存在性严重依赖于网络拓扑的性 
质. 对类似 $\mathrm{BA}$ 的无标度网络, 我们提出的 (EBC, EFR) 策略是一个高效费比和可扩展的设计; 而对 于真实 ISP 路由器级拓扑和 HOT 模型, 并不存在高效费比和可扩展的设计.

更真实的网络流模型, 对网络设计问题更全面的认识以及相关的发现给网络科学和工程科学提供 了深入的认知, 为低成本的网络设计提供了理论依据. 此外, 本文也指出了一些新的研究方向: 1) 在该 框架下, 评价其他设计方案 (如动态路由算法) 的性能; 2) 确定可行解空间的确切形状, 例如, 对不同 的网络, 边界是凹的还是凸的; 3) 在该框架中包含更真实的流量需求; 4) 使用边重连 [43,44] 在图空间 中找出在高效费比设计方面最优的网络拓扑.

致谢 作者感谢程苏琦提供的真实 ISP 路由器级的拓扑.

\section{参考文献}

1 Huston G. Analyzing the Internet's BGP routing table. Internet Protocol J, 2001, 4

2 Broido A, Nemeth E, Claffy K. Internet expansion, refinement and churn. Eur Trans Telecommun, 2002, 13: 33-51

3 Network Science and Engineering (NetSE) Research Agenda. http://www.cra.org/ccc/docs/NetSE-Research-Agenda. pdf, 2009

4 Barabási A L, Albert R. Emergence of scaling in random networks. Science, 1999, 286: 509-512

5 Faloutsos M, Faloutsos P, Faloutsos C. On power-law relationships of the Internet topology. In: Proceedings of ACM SIGCOMM 1999, Cambridge, 1999

6 Newman M E J. Assortative mixing in networks. Phys Rev Lett, 2002, 89: 208701

7 Dorogovtsev S N. Clustering of correlated networks. Phys Rev E, 2004, 69: 027014

8 Zhang G Q, Quoitin B, Zhou S. Phase changes in the evolution of the IPv4 and IPv6 AS-level Internet topologies. Comput Commun, 2011, 34: 649-657

9 Albert R, Barabási A L. Statistical mechanics of complex networks. Rev Mod Phys, 2002, 74: 47-97

10 Dorogovtsev S N, Mendes J F F. Evolution of networks. Adv Phys, 2002, 51: 1097-1187

11 Newman M E J. The structure and function of complex networks. SIAM Rev, 2003, 45: 167-256

12 Zhang G Q, Zhang G Q, Cheng S Q, et al. Symbiotic effect: a guideline for network modeling. Europhys Lett, 2009, 87: 68002

13 Shen H W, Cheng X Q, Cai K, et al. Detect overlapping and hierarchical community structure in networks. Physica A, 2009, 388: 1706-1712

14 Shen H W, Cheng X Q, Fang B X. Covariance, correlation matrix and the multiscale community structure of networks. Phys Rev E, 2010, 82: 016114

15 Cheng X Q, Ren F X, Zhou S, et al. Triangular clustering in document networks. New J Phys, 2009, 11: 033019

16 Newman M E J. Finding and evaluating community structure in networks. Phys Rev E, 2004, 69: 026113

17 Kim K, Kahng B, Kim D. Jamming transition in traffic flow under the priority queueing protocol. Europhys Lett, 2009, 86: 58002

18 Sreenivasan S, Cohen R, López E, et al. Structural bottlenecks for communication in networks. Phys Rev E, 2007, 75: 036105

19 Martino D D, Asta L D, Bianconi G, et al. A minimal model for congestion phenomena on complex networks. J Stat Mech, 2009, P08023

20 Martino D D, Asta L D, Bianconi G, et al. Congestion phenomena on complex networks. Phys Rev E, 2009, 79: 015101

21 Echenique P, Gómez-Gardeñes J, Moreno Y. Improved routing strategies for Internet traffic delivery. Phys Rev E, 2004, 70, 056105

22 Echenique P, Gómez-Gardeñes J, Moreno Y. Dynamics of jamming transitions in complex networks. Europhys Lett, 2005, 71: 325-331 
23 Gupte N, Singh B K. Role of connectivity in congestion and decongestion in a communication network. Euro Phys J B, 2006, 50: 227-230

24 Gupte N, Singh B K, Janaki T M. Networks: structure, function and optimisation. Physica A, 2005, 346: 75-81

25 Zhang G Q, Wang D, Li G J. Enhancing the transmission efficiency by edge deletion in scale-free networks. Phys Rev E, 2007, 76: 017101

26 Yan G, Zhou T, Hu B, et al. Efficient routing on complex networks. Phys Rev E, 2006, 73: 046108

27 Zhao L, Lai Y C, Park K, et al. Onset of traffic congestion in complex networks. Phys Rev E, 2005, 71: 026125

28 Danila B, Yu Y, Marsh J A, et al. Optimal transport on complex networks. Phys Rev E, 2006, 74: 046106

29 Zhang G Q. On cost-effective communication network designing. Eruophys Lett, 2010, 89: 38003

30 Arrowsmith D K, Mondragón R J, Woolf M. Data traffic, topology and congestion. In: Complex Dynamics in Communication Networks. Berlin/Heidelberg: Springer Press, 2005

31 Arenas A, Guilera A D, Guimerra R. Communication in networks with hierarchical branching. Phys Rev Lett, 2001, 86: 3196-3199

32 Borgatti S P. Centrality and network flow. Soc Netw, 2005, 27: 55-71

33 Goh K I, Kahng B, Kim D. Universal behavior of load distribution in scale-free networks. Phys Rev Lett, 2001, 87: 278701

34 Guimerá R, Guilera A D, Redondo F V, et al. Optimal network topologies for local search with congestion. Phys Rev Lett, 2002, 89: 328170

35 Freeman L C. Centrality in social networks: conceptual clarification. Soc Netw, 1979, 1: 215-239

36 Labovitz C, Malan G R, Jahanian F. Internet routing instability. IEEE/ACM Trans Netw, 1998, 6: 515-528

37 Paxson V. End-to-end routing behavior in the Internet. ACM SIGCOMM Comput Commun Rev, 2006, 36: 41-56

38 Li L, Alderson D, Willinger W, et al. A first-principles approach to understanding the Internet's router-level topology. In: Proceedings of ACM SIGCOMM 2004, Portland, 2004

39 Erdös P, Rényi A. On random graphs. Publ Math Debrecen, 1959, 6: 290-297

40 Watts D J, Strogatz S H. Collective dynamics of small-world networks. Nature, 1998, 393: 440-442

41 Spring N, Mahajan R, Wetherall D. Measuring ISP topologies with Rocketfuel. In: Proceedings of ACM SIGCOMM 2002, Pittsburgh, 2002

42 Chiaraviglio L, Mellia M, Neri F. Reducing power consumption in backbone networks. In: Proceedings of IEEE ICC 2009, Dresden, 2009

43 Milo R, Shen-Orr S, Itzkovitz S, et al. Network motifs: simple building blocks of complex networks. Science, 2002, 298: $824-827$

44 Zhang G Q. Traversability of graph space with given degree sequence under edge rewiring. Electron Lett, 2010, 46: $351-352$

\title{
Communication network designing: transmission capacity, cost and scalability
}

\author{
ZHANG GuoQiang ${ }^{1,2 *} \&$ ZHANG GuoQing ${ }^{2}$ \\ 1 School of Computer Science and Technology, Nanjing Normal University, Nanjing 210046, China; \\ 2 Institute of Computing Technology, Chinese Academy of Sciences, Beijing 100190, China \\ *E-mail: guoqiang@ict.ac.cn
}

\begin{abstract}
Despite the growing number of works centering around the traffic dynamics on complex networks, these researches still have some common shortcomings, e.g., too simple traffic flow model and lack of considerations for the designing cost and scalability issues. This paper builds on a more realistic traffic flow model, and offers a holistic view on the network designing problem. In addition to the extensively studied transmission capacity, this
\end{abstract}


paper takes designing cost and scalability as two other designing objectives, and presents a quantitative study of how different designing choices independently and collectively influence these objectives by the introduction of a cartesian coordinate system. It is shown that different kinds of network topologies display different shapes of achievable solution spaces and exhibit different abilities to achieve cost-effective and scalable designing. In particular, we find the philosophy underlying empirical network designing and engineering today fails to meet the cost-effective and scalable designing requirements, and propose a cost-effective and scalable designing scheme for BA-like networks, i.e., the efficient routing combined with effective betweenness based link bandwidth allocation. In addition, when designing a thoroughly new network from the beginning, we find that ER network is a good candidate to achieve cost-effective and scalable designing in most settings.

Keywords cost-effective, communication network designing, network transmission efficiency, network topology, low cost information technology, next generation network, scalable network designing

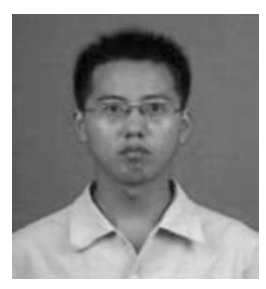

ZHANG GuoQiang was born in 1980 He received the Ph.D degree in computer science from Institute of Computing Technology, Chinese Academy of Sciences, Beijing in 2008. Currently, he is an Associate Professor at School of Computer Science and Technology, Nanjing Normal University. His research interests include network science, future network. Dr. Zhang is a senior member of China Computer Federation.

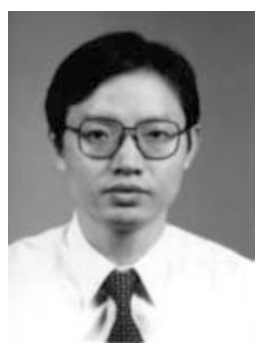

ZHANG GuoQing was born in 1965. He received the Ph.D degree in computer science from Institute of Computing Technology, Chinese Academy of Sciences, Beijing in 2009. Currently, he is an Associate Professor at Institute of Computing Technology, Chinese Academy of Sciences. His research interests include network science and computer networking. 